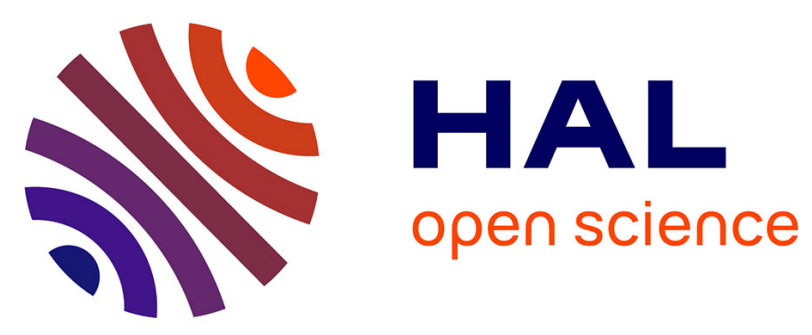

\title{
Polyradiculonévrite aiguë et myopéricardite durant une primo-infection à cytomégalovirus chez un patient sans déficit immunitaire identifié
}

Yohan N'Guyen, Salem Ould Zein, David Petitpas, Patrick Chillet, Philippe Berger, Roland Jaussaud

\section{To cite this version:}

Yohan N'Guyen, Salem Ould Zein, David Petitpas, Patrick Chillet, Philippe Berger, et al.. Polyradiculonévrite aiguë et myopéricardite durant une primo-infection à cytomégalovirus chez un patient sans déficit immunitaire identifié. La Presse Médicale, 2010, 39 (11), pp.1220-1223. 10.1016/j.lpm.2010.06.011 . hal-03267214

\author{
HAL Id: hal-03267214 \\ https://hal.science/hal-03267214
}

Submitted on 28 Jun 2021

HAL is a multi-disciplinary open access archive for the deposit and dissemination of scientific research documents, whether they are published or not. The documents may come from teaching and research institutions in France or abroad, or from public or private research centers.
L'archive ouverte pluridisciplinaire HAL, est destinée au dépôt et à la diffusion de documents scientifiques de niveau recherche, publiés ou non, émanant des établissements d'enseignement et de recherche français ou étrangers, des laboratoires publics ou privés. 
archives-ouvertes

\section{Polyradiculonévrite aiguë et myopéricardite durant une primo-infection à cytomégalovirus chez un patient sans déficit immunitaire identifié}

Yohan N'Guyen, Salem Ould Zein, David Petitpas, Patrick Chillet, Philippe Berger, Roland Jaussaud, Madame Monsieur

\section{To cite this version:}

Yohan N'Guyen, Salem Ould Zein, David Petitpas, Patrick Chillet, Philippe Berger, et al.. Polyradiculonévrite aiguë et myopéricardite durant une primo-infection à cytomégalovirus chez un patient sans déficit immunitaire identifié. La Presse Médicale, Elsevier Masson, 2010, 39, pp.1217 - 1220. 10.1016/j.lpm.2010.05.021 . hal-03267214

\section{HAL Id: hal-03267214 \\ https://hal.archives-ouvertes.fr/hal-03267214}

Submitted on 28 Jun 2021

HAL is a multi-disciplinary open access archive for the deposit and dissemination of scientific research documents, whether they are published or not. The documents may come from teaching and research institutions in France or abroad, or from public or private research centers.
L'archive ouverte pluridisciplinaire $\mathbf{H A L}$, est destinée au dépôt et à la diffusion de documents scientifiques de niveau recherche, publiés ou non, émanant des établissements d'enseignement et de recherche français ou étrangers, des laboratoires publics ou privés. 
int 0.0

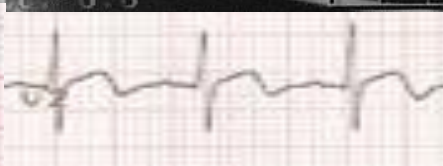

07 sept 13
01 oct 20 
Titre :

Polyradiculonévrite aigüe et myopéricardite durant une primoinfection CMV chez un patient sans déficit immunitaire identifié.

Title :

Cytomegalovirus primary infection with polyradiculoneuritis and myopericarditis in a patient without known immunodeficiency.

Auteurs :

Yohan N'Guyen ${ }^{1,2}$, Salem Ould Zein ${ }^{1}$, David Petitpas ${ }^{1}$, Patrick Chillet ${ }^{1}$, Philippe Berger $^{1}$, Roland Jaussaud ${ }^{2}$.

${ }^{1}$ Service de Réanimation polyvalente, Hôpital de Chalons en Champagne, 51 rue du Commandant Derrien, 51000, Chalons en Champagne, France.

${ }^{2}$ Service de Médecine Interne et Maladies infectieuses, Hôpital Robert Debré, Avenue général Koenig, 51100, Reims, France.

Caractères : 10654 (document entier), 9686 (sans page de garde) . auteur correspondant :

Yohan N'Guyen. Service de Médecine Interne et Maladies infectieuses, Hôpital Robert Debré, Avenue général Koenig, 51100, Reims, France

Mail : yohan.nguyen@wanadoo.fr

Remerciements : aucun.

Support financier : aucun.

Conflits d'intérêts : aucun. 


\section{Introduction :}

Les infections graves à Cytomégalovirus (CMV) de l'immunocompétent sont de plus en plus fréquemment rapportées [1]. Les myocardites à CMV étaient le fait des immunodéprimés [2], mais des observations chez l'immunocompétent ont été décrites [3].

Le CMV est aussi le deuxième agent en cause dans les polyradiculonévrites (PRN) aigües après Campylobacter jejuni [4]. Nous rapportons le cas d'une primoinfection grave à CMV associant une myopéricardite et une PRN aiguë chez un patient sans déficit immunitaire identifié.

\section{Observation:}

Un homme de 48 ans, sans antécédents, est hospitalisé en réanimation pour une tétraparésie fébrile $(\mathrm{J} 1)$. Il présentait depuis 17 jours une fièvre vespérale avec myalgies et toux sèche, auxquelles se sont ajoutées des paresthésies des extrémités depuis 9 jours. L'examen initial retrouvait un patient fébrile à $38^{\circ} \mathrm{C}$ avec une paralysie faciale droite, un globe vésical, des troubles objectifs de la sensibilité superficielle et profonde ainsi qu'une tachycardie et une hypertension artérielle méconnue évocatrices d'une dysautonomie. Les reflexes ostéotendineux étaient abolis. La ponction lombaire initiale était normale sans dissociation albumino-cytologique. Le reste du bilan biologique retrouvait un syndrome mononucléosique et une cytolyse hépatique à $2 \mathrm{~N}$ avec élévation des gamma GT à $4 \mathrm{~N}$. La protéine $\mathrm{C}$ réactive était à $40 \mathrm{mg} / \mathrm{l}$ et la procalcitonine inférieure à $0.5 \mathrm{mg} / \mathrm{ml}$.

Suite à une dégradation de son état neurologique, le patient bénéficie d'une ventilation mécanique à J6 d'abord sur sonde d'intubation puis sur trachéotomie. Le diagnostic de PRN aiguë a été retenu devant la présentation clinique initiale, l'évolution et une dissociation albumino-cytologique (protéinorachie $1,08 \mathrm{~g} / \mathrm{l}$ pour 1 
leucocyte $/ \mathrm{mm}^{3}$ ) sur la seconde ponction lombaire réalisée à J14. Un traitement par immunoglobulines polyvalentes $(0,4 \mathrm{~g} / \mathrm{kg} / \mathrm{j})$ a été entrepris de $\mathrm{J} 4$ à 8 (après 48 heures d'échanges plasmatiques).

Le bilan étiologique de cette PRN (sérologies Campylobacter, VIH, grippe, intracellulaires, cultures virales gorges et selles...) était négatif. Seules une sérologie CMV précoce (J2) et une antigènémie pp65 plus tardive (J15) étaient positives : respectivement, titre en immunoglobulines $\mathrm{G}(\mathrm{IgG}) 67$ pour une norme inférieure à 15 , titre en immunoglobulines $\mathrm{M}(\operatorname{IgM})$ 2,58 pour une norme inférieure à 0,9 et antigènémie CMV positive à 7 noyaux. La polymerase chain reaction Herpes viridae sur le second liquide céphalorachidien était négative. Aucun sérum antérieur n'était disponible et la sérologie CMV de contrôle n'a pas été réalisée. Le diagnostic de PRN dans le cadre d'une primo-infection à CMV a été retenu.

Une antibiothérapie probabiliste par pipéracilline/tazobactam, amikacine et vancomycine a été instituée à J8 dans l'hypothèse d'une pneumopathie nosocomiale sous ventilation mécanique (figure1). Le patient est resté fébrile et tous les prélèvements bactériologiques et virologiques, y compris sur lavage bronchioloalvéoalaire, négatifs.

Une échocardiographie transthoracique (ETT), initialement réalisée à J10 pour éliminer une endocardite, retrouvait un épanchement péricardique de faible abondance associé à un ventricule gauche modérément dilaté, avec baisse de la fraction d'éjection ventriculaire à 58\% et épaisseur normale du myocarde. Les cavités droites et l'oreillette gauche n'étaient pas dilatées, les valves étaient normales. Cet aspect échocardiographique était en faveur d'une myopéricardite. Des troubles diffus de la repolarisation qui n'existaient pas sur l'électrocardiogramme (ECG) initial sont apparus secondairement (figure1).

A J15, un fond d'oeil retrouvait au niveau de l'oeil droit, des hémorragies 
rétiniennes et des nodules dysoriques péripapillaires compatibles avec une rétinite à CMV. Un traitement par ganciclovir est institué à la place de la triple antibiothérapie. L'évolution a été lentement favorable avec amélioration de la radiographie pulmonaire à $\mathrm{J} 21$, normalisation de la fraction d'éjection à $\mathrm{J} 28$, négativation de l'antigènémie à $\mathrm{J} 30$, récupération motrice à partir de J41 et normalisation du fond d'oeil à J48.

L'apyrexie définitive n'est survenue qu'à J49 après traitement d'une infection de cathéter central à Pseudomonas aeruginosa. Le ganciclovir a été relayé par voie orale à J62 et le patient décanulé à J63 après 15 jours de sevrage de la ventilation mécanique. La marche n'est récupérée complètement qu'après 4 mois de convalescence. Le ganciclovir est interrompu sans récidive fébrile à J188. Aucun déficit immunitaire n'a été retrouvé.

Discussion :

Nous présentons le cas d'une primo-infection CMV se manifestant par une PRN associée à une myocardite, une rétinite et une pneumopathie bilatérale chez un patient sans déficit immunitaire identifié. Nous n'avons pas la preuve de la présence du CMV dans chacun des organes atteints mais la présence d'IgM anti CMV et d'une antigènémie CMV positive permet raisonnablement d'imputer l'ensemble des symptômes à la réplication du CMV, tout comme la résolution des différentes atteintes organiques sous ganciclovir. En l'absence de sérum antérieur pour confirmer la séroconversion IgG, en l'absence de sérum de contrôle pour une éventuelle augmentation des titres d'IgG et en l'absence de test d'avidité des IgG, on ne peut prouver formellement qu'il s'agit d'une primoinfection. Cette hypothèse semble plus probable qu'une réinfection à CMV après une primo-infection silencieuse. L'hypothèse d'une réactivation d'une infection CMV ancienne n'a pas été retenue devant l'absence d'immunosuppression. 
Les manifestations organiques dans le cadre des infections à CMV de 
Des études complémentaires sont nécessaires afin de mieux définir les

indications du ganciclovir et les modalités d'une surveillance cardiaque adaptée durant les primo-infections graves à CMV du sujet immunocompétent. 


\section{Bibliographie :}

1- Rafailidis P, Mourtzoukou E, Varbobitis I, Falagas M. Severe cytomegalovirus infection in apparently immunocompetent patients : a systematic review. Virol J 2008;5:47.

2- Millett R, Tomita T, Marshall HE, Cohen L, Hannah H. Cytomegalovirus endomyocarditis in a transplanted heart. A case report with in situ hybridization. Arch Pathol Lab Med. 1991;115(5):511-5.

3-Fernández-Ruiz M, Muñoz-Codoceo C, López-Medrano F, Faré-García R, CarbonellPorras A, Garfia-Castillo C et al. Cytomegalovirus myopericarditis and hepatitis in an immunocompetent adult: successful treatment with oral valganciclovir.

Intern Med 2008;47(22):1963-6.

4- Hughes RA, Hadden RDM, Gregson NA, Smith KJ. Pathogenesis og Guillain Barré syndrome.

J Neuroimmunol 1999;100:94-7.

5-Arnold AG, Lawrence DS, Corbitt G. Cytomegalovirus and the Guillain Barré syndrome.

Postgrad Med J. 1978;54(628):112-4.

6- Kytö V, Vuorinen T, Saukko P, Lautenschlager I, Lignitz E, Saraste A et al. Cytomegalovirus infection of the heart is common in patients with fatal myocarditis. Clin Infect Dis. 2005;40(5):683-8.

7- Steininger C. Clinical relevance of cytomegalovirus infection in patients with disorders of the immune system.

Clin Microb Infect 2007;13:953-963.

8- Krmpotic A, Bubic I, Polic B, Lucin P, Jonjic S. Pathogenesis of murine cytomegalovirus infection. 
Microbes Infect 2003;5:1263-1277.

9- Kim YS, Hollander H. Polyradiculopathy due to cytomegalovirus: report of two cases in which improvement occurred after prolonged therapy and review of the literature. Clin Infect Dis. 1993;17(1):32-7. 
Figure 1 : iconographie.

1

2

3

4

5

6

7

8

9

10

11

12

13

14

15

16

17

18

19

20

21

22

23

24

25

26

27

28

29

30

31

32

33

34

35

36

37

38

39

40

41

42

43

44

45

46

47

48

49

50

51

52

53

54

55

56

57

58

59

60

61

62

63

64

65

en haut : radiographie et scanner montrant la pneumopathie bilatérale prédominant à droite (J8).

en bas à gauche : dérivations précordiales de l'électrocardiogramme d'entrée (J3).

en bas à droite : dérivations précordiales de l'électrocardiogramme réalisé au moment de la myopéricardite (J10). 
Réponse aux commentaires sur le manuscrit : Polyradiculonévrite aigüe et myopéricardite durant une primoinfection CMV chez un immunocompetent. No LPM-D-10-00241

Madame Monsieur,

Veuillez recevoir les réponses à toutes les suggestions que vous m'avez fait parvenir.

Reviewer 1:

Tout à fait d'accord avec vous, je ne l'ai peut être pas bien exprimé mais la question que je posais est de l'intérêt du ganciclovir dans les primoinfections graves de l'immunocompétent. Il n'est pas légitime de proposer un tel traitement dans des infections bénignes spontanément résolutives : le rapport bénéfice risque serait bien moins favorable alors. Je corrige la page 5 afin de retirer toutes les phrases portant à confusion.

Reviewer 2 :

Introduction P2: D'accord, mais il faut garder à l'esprit le patient dont nous présentons l'observation n'a pas présenté qu'un syndrome de Guillain Barré isolé (cf réponse infra).

Rédaction du cas clinique : je suis d'accord pour reformuler en plus condensé pour que les informations que vous souhaitez voir en priorité sautent plus aux yeux. Je souhaite par contre laisser tout le descriptif clinique et biologique car pour ce patient le diagnostic de PRN, myocardite et rétinite sur primoinfection à CMV est retenu devant :

- l'argument de fréquence du CMV comme vous le disiez en tant que second agent en cause dans les PRN

- une sérologie positive avec IgM et antigénémie, (ce qui peut être banal en réanimation)

- en l'absence de séroconversion documentée (pas de sérum antérieur), en l'absence d'avidité et en l'absence d'ascencsion du titre à J15 car sérologie non faite du fait des tégélines.

- et retenant le diagnostic de primoinfection bruyante plus probable qu'une réactivation bruyante après une primoinfection silencieuse chez un patient non immunodéprimé.

Il est à mon sens vraisemblable que le diagnostic soit bien celui là mais je souhaite laisser tous ces éléments pour que le lecteur puisse se faire son opinion et n'ait pas l'impression qu'on lui cache quelque chose dans ce contexte où le diagnostic biologique est attaquable.

L'EMG n' a pas été réalisé pour ce patient (ce sont les aléas de la «vraie vie », la seule neurologue qui savait lire les EMG dans cet hopital périphérique était en vacances à cette période là de l'été). Il y'avait effectivement un intérêt initial à le réaliser mais le diagnostic de PRN a été retenu sur la clinique initiale, la dissociation albumino-cytologique lors de la deuxième PL et l'évolution.

D'accord.

P3 : Ok pour simplifier

L'Echocardiographie a été réalisée initialement pour éliminer une endocardite devent cette fièvre prolongée en réanimation chez un patient porteur d'un cathéter central. Je rajoute dans le texte.

J'ai par contre précisé volontairement le switch de l'antibiothérapie vers le ganciclocir seul pour souligner que l'antibiothérapie à large spectre n'avait aucun effet sur la fièvre alors que sous 
ganciclovir (ou spontanément) le patient s'est mis en apyrexie et a corrigé une à une toutes les atteintes organiques décrites dans l'observation. Cette précision permet d'exclure tout rôle de l'antibiothérapie dans l'évolution du patient.

P4 : Non, effectivement on ne peut affirmer de manière indéniable que toutes les atteintes organiques (rétinite, myocardite, pneumopathie) soient liées au CMV, en l'absence d' effet cytopathogène du virus identifié par exemple sur une biopsie d'organe (ex biopsie endomyocardique). C'est le sens de la phrase : « [...] Nous n'avons pas la preuve de la présence du $C M V$ dans chacun des organes atteints mais la présence d'IgM anti CMV et d'une antigènémie $C M V$ positive permet raisonnablement d'imputer l'ensemble des symptômes à la réplication du $C M V$, tout comme la résolution des différentes atteintes organiques sous ganciclovir. »

Il paraît somme toute moins probable d'avoir 4 maladies différentes (une pour la rétinite, une pour la myocardite, une pour la pneumopathie et une pour la PRN) que d'avoir un seul diagnostic uniciste chez un sujet jeune sans antécédents. De plus, cette démarche probabiliste correspond davantage à la réalité : on ne peut prendre le risque de déplacer un malade intubé/ventilé pour réaliser une biopsie pulmonaire ou une biopsie endomyocardique sans bénéfices clairs derrière sur le plan thérapeutique, alors qu'un traitement d'épreuve a un meilleur rapport bénéfice/risque.

On a donc considéré de ce fait que ce malade avait une atteinte polyviscérale due au CMV, et nous l'avons mis sous Ganciclovir. Il n'y a en effet aucune preuve de l'efficacité du ganciclovir dans les PRN ni dans les atteintes polyviscérales du CMV chez le patient non immunodéprimé, mais l'intérêt de cette observation est de le discuter et je citerai l'autre reviewer de ce manuscrit dont je partage le point de vue : «[...] je doute que l'on puisse évaluer correctement l'intérêt des anti CMV dans l'infection à CMV de l'immunocompétent. Mais compte tenu de l'efficacité in vitro et de l'efficacité chez l'immunodéprimé, on devrait conclure que toute forme sévère d'infection à CMV même chez un immunocompétent devrait conduire à prescrire un anti CMV, ganciclovir ou foscarnet. »

Comité de rédaction :

D'accord avec votre suggestion de prudence. Je change le titre, la dernière phrase de l'introduction page 2 et la discussion page 5 . Je me suis par contre permis de retirer primitif.

Veuillez agréer Madame, monsieur, l'expression de mes salutations distinguées.

Yohan N'Guyen 